\title{
A Novel Algorithm for Fire/Smoke Detection based on Computer- Vision
}

\author{
Jean Paul Dukuzumuremyi ${ }^{1}$, Beiji Zou ${ }^{2}$ and Damien Hanyurwimfura ${ }^{3}$ \\ ${ }^{1,2}$ College of Information Science and Engineering, Central South University, \\ Changsha, 410083, China \\ ${ }^{3}$ College of Information Science and Engineering, Hunan University, Changsha, \\ 410082, China \\ dukuze@gmail.com,bjzou@vip.163.com,hadamfr@gmail.com
}

\begin{abstract}
The scarcity of automatic fire detection systems continues to be a problem that needs a serious attention in order to save human lives by preventing injuries ind/or deaths. The newest innovations are continuing to use cameras and computer algorithms to analyze the visible effects of fire and its motion in their applications. As their approaches present some drawbacks when working in spatial domain, the main difficuly is still to identify objects if they do not occur at the expected position. In this paper, we present an improved fast and robust algorithm for detecting fire/smoke in a clutteredscene from a pair of cameras. The input images are first segmented according to a predetermined disparity threshold map and the real-time disparities of fire. Binary image processing techniques are used to reject noise introduced into the segmented images through 6 w-resolution disparity calculations which consequently can lead to the gain of clearer kesults. In order to reduce the false alarms, a new segmentation method used in this approach shows that segmented images using stereovision are more accurate than those segmented using color approach for the overall detection. The segmented images are then used for image feature extraction for a Fuzzyneural network classifier to help the ystem to generate a warning in case fire/smoke is detected.
\end{abstract}

Keywords: Stereo vision. fire detection, fuzzy neural network, segmentation

\section{Introduction}

Fire accidents frequently put in danger our life at the same time causing economical and ecological darmages and the scarcity of automatic fire detection systems continues to be a problem that really needs a serious attention to save human lives by preventing injuries and/or deaths (To avoid the fire disasters, many early fire-detection techniques have been explored and most of them are based on particle sampling, temperature sampling, relative humidity sampling, air transparency testing, smoke analysis, in addition to the traditional ultraviolet and infrared fire detectors. However, those detectors either must be set in the proximity of a fire or can't provide the additional information about the process of burning, such as fire location, size, growing rate, and so on. Thus, they are not always reliable because energy emission of non-fires or by products of combustion, which can be yielded in other ways, may be detected by misadventure. This usually results in false alarms. To provide more reliable information about fires, Stereo Vision-based approach is becoming more and more helpful. The newest innovations are continuing to use cameras and computer algorithms to analyze the 
visible effects of fire and its motion in their applications. As their approaches present some drawbacks when working in spatial domain, the main difficulty is still to identify objects if they do not occur at the expected position. We propose stereo vision segmentation system that can first segment the input images in comparison with the pre-existing disparity threshold map and the real time disparities of the odd situation. Binary image processing techniques are used to reject noise introduced into the segmented images through low-resolution disparity calculations. The segmented images are then used for image features extraction for a neural network classifier.

\section{Related Works}

Conventional point-type thermal and smoke detectors are widely used nowadays but they typically take charge of a limited area in space. In large rooms and high buildings, it may take a long time for smoke particles and heat to reach a detector. In this work we preferred a video based fire detection (VFD) which is a newly developed teehn que as it can greatly complete the fire detection requirements in large rooms and high buildings, and even for outdoor environment [1]. Up to now, most of methols nake use of the visual features of fire including color, textures, geometry, flickering and motion, [2, 3]. Some researchers used RGB input as a simple and effective procedure for real-time application. To improve the RGB systems, a fuzzy logic enhanced/generic color model for fire pixel classification is proposed. The model uses YCbCrcolor space to separate the luminance from the chrominance more effectively thaneolor spaces such as RGB as studied in [4.] An algorithm of early firre image detection and identification based on discrete fractal brownian incrementabrandom fieldmodel is proposed in [5]. It is important to realize that most of fire detection systems used the heuristic fixed threshold values in their specific methods as did in [6.]. In addition to motion and color clues used in the literature reviewed above another approach proposing smoke/fire detection by analyzing the video in wavelet domain has been treated in [7]. To improve the solutions of the shortcomings enumerated above, many researchers opted computer vision based fire/smoke detection. Some techniques such as combination of color and stereo vision used in $11,7,8]$ have been developed to compensate small or gradual changes in the scene or the lighting. Although stereo vision and edge detection proposed in 9] can be used to detect independently moving targets in the presence of camera motion, jt is not feasible for no-rigid object extraction since the movements of the fire/smoke are different. To increase reliability, some systems like the one used in $[8,10]$, integrate multiple cues such as stereo, color, wavelet pattern to detect fire /smoke. However, color is very sensitive to illumination changes [10]. These systems prove that stereo and wavelet are more reliable and helpful cues than color and wavelet fire detection in general situations. To overcome the shortcomings listed above, after segmenting an image, we have extracted some features that allow fuzzy-neural network to easify detect features of the fire/smoke by analyzing the image using DWT and DCT [11]

\section{Proposed Approach}

Our new fire warning system is designed to be equipped with a stereo-based object detection module that detects foreground objects in real-time based on disparity map segmentation, and its main function is to improve the previous work in reducing false fire alarms by distinguishing fire information from other objects present in the scene. This is achieved through stereo-based segmentation, wavelet transform and discrete cosine for features extraction and recognition based on fuzzy-neural networks. The fuzzy-neural 
network modeling system is developed to model a system with some descriptive fuzzy logic rules that are designed to deal with complex, ill-defined and unconstrained problems. The two main purposes of fuzzy logic are; (i) to make an accurate and quick procedure to approach the desired result; (ii) to build a general and flexible mechanism which is applicable in solving various fields of modeling problems shown in [12, 13]. As accuracy is very important in classifiers used for emergency applications, a high percentage of false negatives in screening systems increases the risk of real fire images not receiving the attention they need, while a high false alarm rate causes unwarranted worries and increases the load on system resources.

\section{The algorithm}

Our algorithm consists of four steps: preprocessing, segmentation, features extraction and classification. The preprocessing deals with image enhancement, the segmentation deals with object extraction plus object morphological operation for refinement of the object boundary, the feature extraction is accomplished using wavelet and discrete cosine transforms, lastly we recognize the fire/smoke frame based on fuzzy classification.

\subsection{Dataset and Preprocessing}

We took 1000 images of fire/no fire and smoke/no smoke to use forkesting this method. Every image has been resized to $256 \times 192$. The data set contains eight fire/smoke categories: DWF(Day With Fire), DNF(Day No Fire), NWF(Night Without Fire), NNF(Night No Fire), DWS(Day With Smoke), DNS(Day No Smoke), NWS(Night With Smoke), and NNS(Night No Smoke). Within DNF and NNF, we useed several differentenvironments such as candles, electrical tubes, paper fire and plastic fire in night shots or daytime shots to gain the variability. In order to get variability of baekground we took ON/OFF lights for daytime and night shots. Figure 1 depicts the genefal flowchart of our fire/smoke classification system. The system input is a pair stereo images (Raw Inage and background image). The system output is the class label (from 1:8) to which the fire belongs. First the object is extracted using the proposed technique, and then two image features generators (DCT and DWT) are computed simultaneously on the segmented object, finally a Fuzzy Neural Network (FNN) trained offline is used to classify the objects into one of the eight image categories.

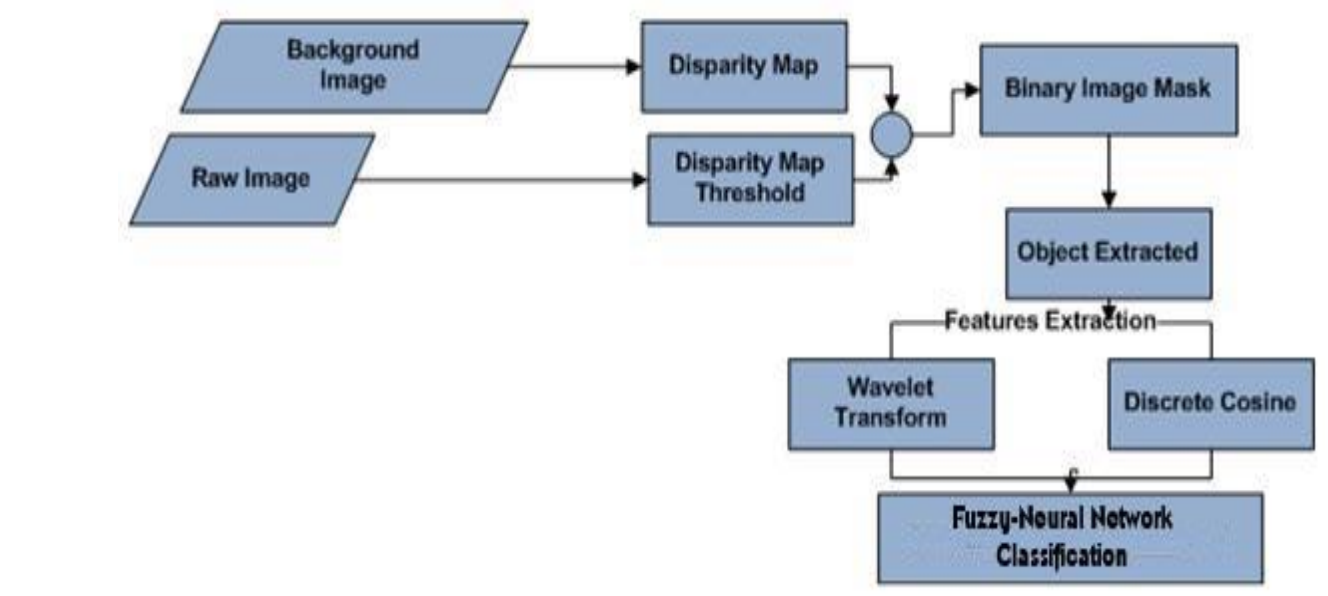

Figure 1. Flowchart of fire/smoke classification. 


\section{Stereo Setup and Segmentation Algorithm}

Given 3D information recovered from the calculation of the disparity map, the main issue to solve is the determination of the lowest boundary of the house interior in the disparity space. Then, the object of interest will be defined as all connected pixels with disparity values larger than the predetermined disparity threshold map, see Figure 2 and Figure 3.

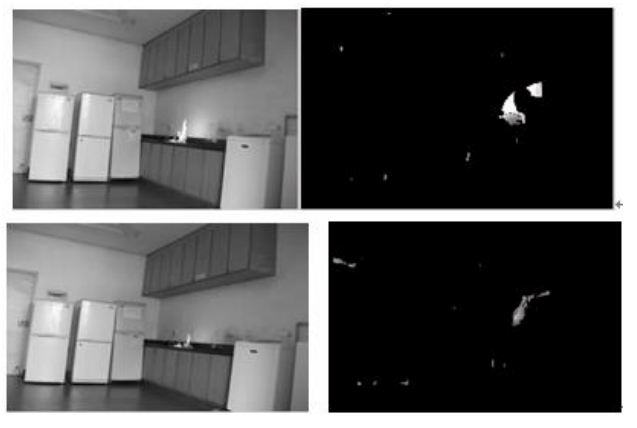

Figure 2. The objects (fire in the kitchen with its, background) and their corresponding disparity maps

(a)
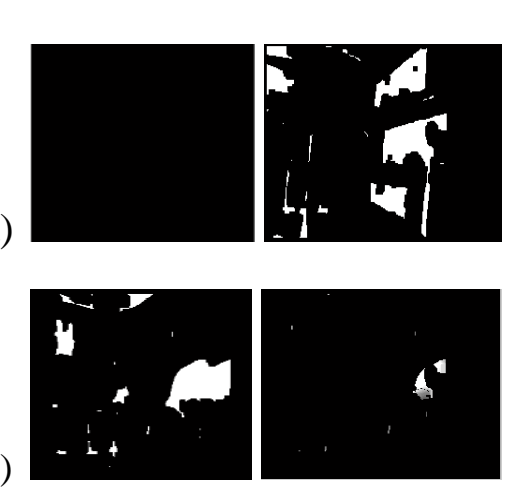

Figure 3. Results of the disparity-based object extraction approach on images in indoor scenes. The rows (a) and (b) show the disparity map of an original image (right column and the extracted object image (left column) of kitchen background, high flame in kitchen, low flame and very low flame, respectively

The proposed algorithm mainly consists of three steps:

1. Off-line determination of a unique disparity threshold map: Establish a predetermined disparity threshold map (disparity template) that defines the background surfaces for suppression. This disparity template is built off-line.

2. Current creation of a binary suppression mask by using the disparity map of the present situation and the pre-determined threshold map. The disparity map of a new pair stereo is generated during the operation and compared with the disparity threshold map. The output is a binary mask. The background pixels are assigned to zeros .

3. Employ morphological closing operator to remove the noise and smooth the foreground regions: due to noise and other artifacts coming from the calculation of the disparity map, the extracted object from the previous step might appear as containing holes or even being sliced into several parts. This refinement stage of the algorithm 
handles these problems through binary image processing techniques including dilation, singularity suppression and erosion.

\subsection{Disparity Threshold Map Determination}

As The disparity threshold map is generated off-line using a set of representative images of the interiors of a kitchen without fire/smoke presence. Both physical and virtual boundary surfaces with added artificial textures to the physical boundary are used. A physical boundary is defined as the interior of the kitchen or a structure that limits the view depth of the cameras. A virtual boundary surface can be established from the stereo camera configuration and the de-sired maximum view depth of the camera. Obviously, the selected boundaries should contain the maximum volume within which a fire/smoke will be classified. For better range accuracy, artificial textures are added to the physical boundary surfaces in creating the disparity threshold map. in order to obtain the perceptual benefits of texture gradients. The goal of this approach is not to produce realistic textured images, but rather to aid the visualization of rendered surfaces. For the same reason, averaged dispanty yalues of a number of image frames are used in the process. Experimentally an average of over 100 frames is good enough to establish the disparity threshold map [11]. After the heading number, not a colon.

\subsection{Background Suppression}

A comparison between the measured disparity value and the disparity threshold for all the pixels in the image creates a binary map ha ing the same matrix correlation as the original image. For a given pixel location $(i, j)$, assume the measured disparity value is $D b(i, j)$ and the disparity threshold is $D t(i, j)$. The new binay map $Q(i, y)$ with the same matrix correlation as the original image is first created with the following rules: If $D b(i, j) \leq D t(i, j)+\mu(i, j)$, then $Q(i, j)=0$ otherwise $Q(i, j)=1$, where the parameter $\mu(i, j)$ is used to control the degree of background suppression. It can be a function of the disparity threshold $D t(i, j)$, the location of the pixel or simply a constant. In our current implementation, it was chosen as a constant for simplicity. The disparity value is defined in such a way that it increases as the object distance decreases. In this case, the areas indicated by $0 s$ in $Q(i$, $j$ ) represent objegts that are beyond the desired detection range. Similarly, the areas indicated by 15 in $Q(i, j)$ represent objects that are within the desired detection range. We have set up the stereo Canneras so that their image planes are embedded within the same plane and separated yith baselength $(b)$ of $10 \mathrm{~cm}$ as shown in figure 4 . Under this condition, the difference between $d l$ and $d r$ is called the disparity, and is directly related to the distance of the object normal to the image plane [1].

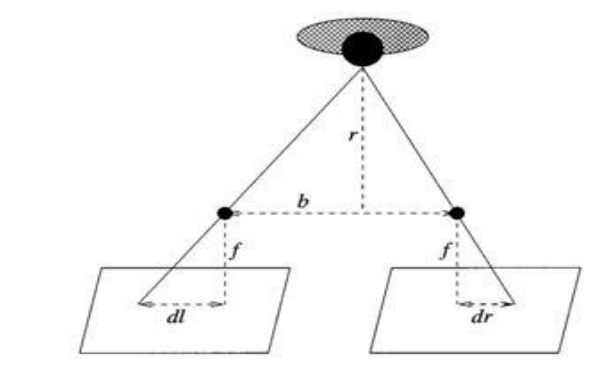

a)

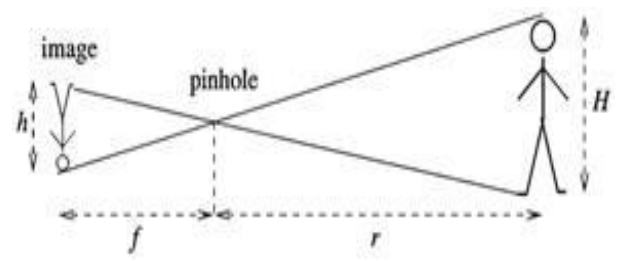

b)

Figure 4. (a) Stereo geometry, (b) image-forming geometry 


\subsection{On-line Morphological Operator Application for Object Enhancement}

In order to reduce the noise effects, fill out the holes and/or connect several parts of the object of interest, binary image processing techniques of type opening and closing operations are used to achieve a clean and accurate background suppression mask. [14].

- A $3 \times 3$-way flat structuring element for morphological operators is applied to the initial binary mask $Q(i, j)$ where two different levels of erosion and other singularity suppression operators are introduced here and applied as follows:

- Erosion Level 1: If the summation of its 8-way neighbors is greater than or equal to 1 , set $Q(i, j)$ to 1 ; otherwise set $Q(i, j)$ to $0[11]$.

- Erosion Level 2: If the product of its 8-way neighbors is 0 , then set $Q(i, j)$ to 0 , otherwise set $Q(i, j)$ to 1 .

- Suppression: If the summation of jts 8 -way neighbors is less than or equal to 3 , set $Q(i, j)$ to 0 , otherwise no change to $Q(i, j)$.

Since the segmentation is based on a low-resolution disparity calculation the disparity calculation is applied block by block instead of pixel by pixel. The block size is $10 \times 16$ pixels. Multiple binary image processing steps should be applied to the new binary map to reject noise.

\section{Features Extraction}

The DCT (Discrete Cosine Transform) and DWT (Discrete Wavelets Transform) have been our features generators. The DCT has been applied to the image block and only the first 100 of the total transformed coefficients are retained after zigzag coding as they store the maximum information of the image as discussed in [15]. For DWT three levels have been applied to the segmented images. In both cases (DCT and DWT), the five features chosen were arithmetic mean, geometric mean, standard deviation, skewness and entropy. In normal (non-fire) images, these features follow a certain pattern which is quite different from images with fire. Similarly, in case of fire-images, the skewness is more towards the left (called left skewness) [12]. However, one single feăture could not classify fire/smoke and non-fire/smoke consistently. Hence all the features were retained.

\section{Fuzzy Logic Based Fire Recognition}

For Multi-Input 5 Single Output (MISO) fuzzy modeling systems, a simple and widely used type of Fuzzy Neural Network (FNN) called Sugeno model is applied in this paper. For sirnplicity, we assume that there are three rules in the fuzzy reasoning system of Sugenomodel.

The rules explained are the result of the tests we carried upon our images and the three different approaches of segmentation (Non-segmentation, K-means segmentation and (D) sparity map Segmentation). Here we have to admit that we were biased on the fact that it is better for the system to make a mistake by alarming a false fire than not alarming a true fire.

With $\mathrm{m}=$ mean, $\mathrm{e}=$ entropy and st.d = standard deviation:

For Non-segmented images:

$\mathrm{R} 1$ : If $\mathrm{m}$ is low, e is low, st.d is high then the image has fire.

$\mathrm{R} 2$ : If $\mathrm{m}$ is medium, e is low, st.d is high then the image has fire.

$\mathrm{R} 3$ : If $\mathrm{m}$ is high, e is high, st.d is medium then the image has fire.

$\mathrm{R} 4$ : If $\mathrm{m}$ is high, e is high, st.d is low then the image has no fire. 


\section{For K-means segmentation:}

$\mathrm{R} 1$ : If $\mathrm{m}$ is high, e is low, st.d is low then the image has fire.

$\mathrm{R} 2$ : If $\mathrm{m}$ is low, e is low, st.d is high then the image has fire.

$\mathrm{R} 3$ : If $\mathrm{m}$ is high, e is low, st.d is medium then the image has fire.

$\mathrm{R} 4$ : If $\mathrm{m}$ is low, e is low, st.d is medium then the image has no fire.

\section{For Disparity map segmentation:}

$\mathrm{R} 1$ : If $\mathrm{m}$ is low, e is low, st.d is low then the image has fire.

$\mathrm{R} 2$ : If $\mathrm{m}$ is low, e is med, st.d is med then the image has fire.

R3: If $\mathrm{m}$ is high, e is high, st.d is high then the image has no fire.

Mean, entropy and standard deviation are the input variables; low, middle and high are fuzzy subsets of the inputs; $f i$ is the conclusion of the fuzzy rule and the function of input variables; Fire and no fire are the results got from the fuzzy classification. The operation of FNN is Gradient descent method. The aim is the minimization of the error function $E=1 / 2$ $(d-y)^{2}$ where, $y$ is the output of the fuzzy logic, $d$ is the expected output of the jth sample. The weight $i_{j}, w$ is trained by the learning rules of equation (1) and (2) [13].

$$
\begin{aligned}
& w_{i j}(t+1)=w_{i j}(t)-\eta \frac{\partial E}{\partial w_{i j}} \alpha \Delta w_{i j}(t) \\
& \Delta w_{i j}(t)-w_{i j}(t-1)
\end{aligned}
$$

Where $\eta$ is the learning velocity, $\alpha(0<\alpha<1)$ is the gradient.

\section{Experiment results}

For machine learning, 1000 images of fire/no fire and smoke/no smoke were used for testing the proposed method. Every image has been resized to $256 \times 192$. The data set contains eight fire/smoke categories: DWF(Day)With Fire), DNF(Day No Fire), NWF(Night Without Fire), NNF(Night No Fire), DWS(Day With Smoke), DNS(Day No Smoke), NWS(Night With Smoke), and NNS(Night No Smoke) Within DNF and NNF, we used several different environments such as candles light, electrical tubes light, paper fire and plastic fire in night shots and day time shots to gain the variability.

In order to get variability of background, we took ON/OFF lights for daytime and night shots.

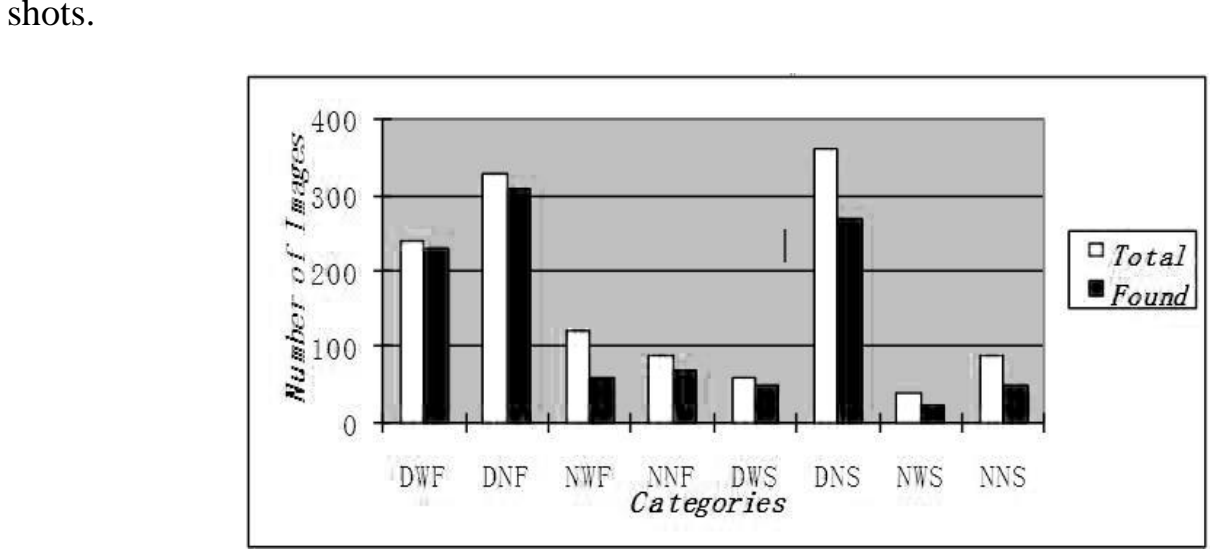

Figure 5. Fire/smoke classification performance: K-means segmentation method 


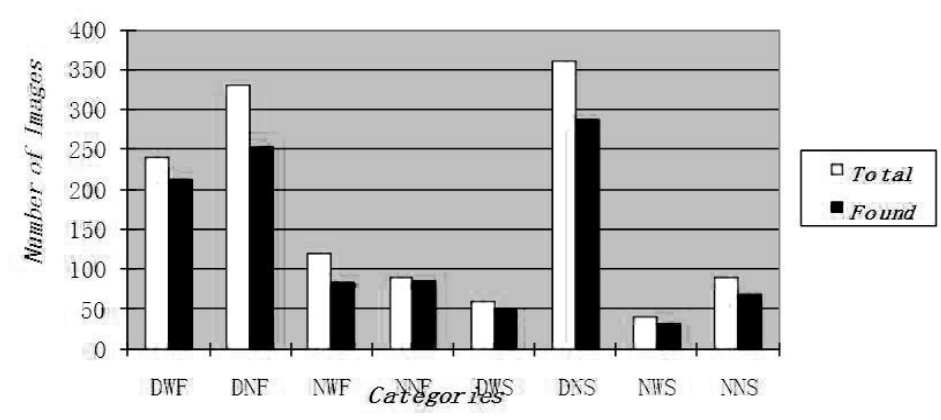

Figure 6. Fire Fire/smoke classification performance: Disparity map segmentation method

As shown in literature review, most fire and flame detection/recognition algorithms are based on color and motion analysis in video. But it is clear that fires gcourring at night have different temporal and spatial characteristics than daytime fires as shown in figure 5 and figure 6, $48.19 \%$ were found from non-segmented images, $79.92 \%$ from k-means segmentation and $80.6 \%$ from disparity map segmentation. Here it can be seen that the overall of both k-means and disparity segmentation is almost the same but when it came to illumination changes and motion, disparity outperforms well as NWF found $70 \%$ versus $50 \%$, NNF $94 \%$ versus $77.77 \%$, NNS $77.6 \%$ versus $60 \%$ and NWS $75.55 \%$ versus $55.55 \%$.

Table 1 shows in different periods of time the comparative results between disparity map segmentation and k-means only; the non segmented images are not taken into account here as the early work made clear of their very low performance [12].

Table 1. Detailed perfor̂mance comparative analysis

\begin{tabular}{|c|c|c|c|c|c|c|}
\hline Class & \multicolumn{3}{|c|}{ Disparity Segmented images } & \multicolumn{3}{|c|}{ K-means-segmented images } \\
\hline & $\begin{array}{c}\text { Accuracy } \\
(\%)\end{array}$ & Sensitivity & Specificity & $\begin{array}{c}\text { Accuracy } \\
(\%)\end{array}$ & Sensitivity & Specificity \\
\hline DWF & 88.8 & & 0.8 & 66.7 & 0.72 & 0.65 \\
\hline $\mathrm{DNF}$ & 91.6 & 0.96 & 0.93 & 80 & 0.89 & 0.62 \\
\hline NWF & 80 & 0.9 & 0.85 & 40 & 0.75 & 0.43 \\
\hline NNF & 75 & 0.88 & 0.66 & 56 & 0.69 & 0.52 \\
\hline DWS & 66.6 & 0.83 & 0.66 & 50 & 0.54 & 0.36 \\
\hline DNS & 92 & 096 & 0.87 & 49 & 0.9 & 0.27 \\
\hline NWS & 57 & 0.75 & 0.87 & 44 & 0.6 & 0.46 \\
\hline NNS & & 0.875 & 0.8 & 42 & 0.69 & 0.39 \\
\hline All & 62.6 & 0.71 & 0.644 & 42.7 & 0.578 & 0.37 \\
\hline
\end{tabular}

The comparison provided in Table 2 shows that the disparity based segmentation is a very promising way to improve the performance of fire/smoke classification. Note that the overall accuracy is rather low due to the fact of the significantly different conditions between the training and testing data set. For every segmentation method mean, entropy and standard deviation were calculated with Matlab tools. Values obtained were classified first on human perception judgment considering interval in which most of the values are concentrated, before being used for the NFL and then the NFL was tested to see whether it could recognize the image for existence or nonexistence of fire. From the 
results it is clear that disparity based segmentation presented an advantage of having fewer rules. To evaluate the three different segmentation methods, accuracy, sensitivity and specificity were used as defined in equation (3), (4) and (5).

Table 2. DCT and WT Performance comparison for the three segmentation methods

\begin{tabular}{l|l|l|l|l|l|l|l|l|l|}
\hline & \multicolumn{3}{|l|}{ Non-segmented } & \multicolumn{3}{l|}{ K-means } & \multicolumn{2}{l|}{ Disparity map } \\
\hline Class & $\begin{array}{l}\text { Accu- } \\
\text { racy(\%) }\end{array}$ & $\begin{array}{l}\text { Sensi- } \\
\text { tivity }\end{array}$ & $\begin{array}{l}\text { Speci- } \\
\text { ficity }\end{array}$ & $\begin{array}{l}\text { Accu- } \\
\text { racy(\%) }\end{array}$ & $\begin{array}{l}\text { Sensi- } \\
\text { tivity }\end{array}$ & $\begin{array}{l}\text { Speci- } \\
\text { ficity }\end{array}$ & $\begin{array}{l}\text { Accu- } \\
\text { racy(\%) }\end{array}$ & $\begin{array}{l}\text { Sensi- } \\
\text { tivity }\end{array}$ & $\begin{array}{l}\text { Speci- } \\
\text { ficity }\end{array}$ \\
\hline DCT & 55 & 0.48 & 0.53 & 82 & 0.70 & 0.65 & 92 & 0.75 & 0.78 \\
\hline WT & 51 & 0.40 & 0.31 & 42.7 & 0.578 & 0.37 & 62.6 & 0.71 & 0.644 \\
Accuracy & $=\frac{T P+T N}{T P+T N+F P+F N} x 100 \%$ \\
Sensitivit & $y=\frac{T P}{T P+F N}$ \\
Specificit & $y=\frac{T N}{T N+F P}$
\end{tabular}

Where $T P$ is the true positive, $T N$ the true negative, $F P$ the false positive, and $F N$ the false negative. The non-segmentation resulted in accuracies of 55 and 51 with DCT and DWT features generator. Sensitivity of 0.75 for DCT and 0.71 for DWT and specificity of 0.78 for DCT and 0.644 of DWT fô disparity segmentation method are higher compared to 0.578 and 0.37 respectivery from the k-means which on its turn has presented better results compare to non segmentation method.

\section{Conclusion}

The algorithm based on computer-vision is proposed for fire/smoke detection. To check the robustness of the used technique, stereo vision segmentation was carried out on indoor fire/snokes frames. The input images are first segmented according to a predetermined disparity chreshold map and the real-time disparities of the fire/smoke. Binary image processing techniques are used to reject noise introduced into the segmented images through low resolution disparity calculations. The system is implemented in Matlab on a Windows PC. The disparity segmentation averaged overall accuracy is about $20 \%$ better than that of the classifier with k-means image segmentation and $27 \%$ of precision better than that of the k-means based segmentation. Sensitivity of 0.73 and specificity of 0.712 resulting from the disparity segmented image classifier have shown that it is snarter than other segmentation methods.

\section{Acknowledgements}

This work is supported by the National Science Foundation of China under Grant No. 60970098, Modeling for Human Visual System and its application; The National Science Foundation of China under Grant No. 61173122, A Fast Three-dimensional Reconstruction Based on Image by Simulating Mechanisms of Human Visual System. 


\section{References}

[1] L. Rossi , M. Akhloufi and Y. Tison , "Dynamic Fire 3D Modeling using a Real-time Stereo Vision System”, Journal of Communication and Computer, vol. 6, no. 10 (2009), pp. 33-38.

[2] C. Turgay, "Fast and Efficiency Method for Fire Detection Using Image Processing", ETRI Journal, vol. 32, (2010), pp.881-890.

[3] J. Chen, Y. Du, and D. Wang, “An Early Fire Image Detection and Identification Algorithm Based on DFBIR Model”, WRI World Congress on Computer Science and Information Engineering (2009), March 312009 April 2, Los Angeles, CA USA, pp. $229-232$.

[4] C. Bo-Ho, B. Jong-Wook and J. Sung-Hwan, "Image Processing-Based Fire Detection System using Statistic Color Model", International Conference on Advanced Language Processing and Web Information Technology, ALPIT '08 (2008), July 23-25, Dalian Liaoning, pp. 245 - 250 .

[5] M. A. Akhloufi, R. Lucile, A. Lilia and T. Yves, "Dynamic Fire Modeling in Three-dimensional Space", First Workshops on Image Processing Theory, Tools and Applications, (2008), November 23-26, Sousse, pp. 1-\$.

[6] K. ByoungChul, H. Kwang HoCheong, and Y. Jae. "Early Fire Detection Algorithm basec on Irregular Patterns of Flames and Hierarchical Bayesian Networks", Fire Safety Journal, vol. 45, no. 4 (2010), pp. $262-$ 270.

[7] M. N. Do, and V. Martin, "Wavelet-Based Texture Retrieval Using Generalized Gaussian Density and Kullback-Leibler Distance”, IEEE Transactions on Image Processing. vol 11, no. 2 (2002), pp. 146 - 158.

[8] T. X. Tung and K. Jong-Myon, "Fire Detection with Video using Fuzzy k-means and Back-propagation Neural Network", Proceedings of the 8th international conference on Advances in nerral networks, vol. Part II, (2011), pp. 373-380.

[9] H. SunJae, K. ByoungChul, and N. JaeYeal, "Fire-Flame Detection based on Fuzzy Finite Automation", International Conference on Pattern Recognition, August 23-26, Istanbul (2010), pp. 3919 - 3922.

[10] G. Jayavardhana, M. Slaven, and P. Marimuthu, "Smoke Detection in Yideo using Wavelets and Support Vector Machines", Fire Safety Journal, Elsevier, vol. 44, no. 8, (2009), pp. 1110-1115.

[11] K. Henry, S. Qin, B. William, S. Kiselewich, A. Paul and H. Rad,' Disparity Based Image Segmentation for Occupant Occupation", Conference on Computer Vision and Pattern Recognition, May 27-June 02, (2004), pp. 126.

[12] D. Jean Paul, U. Marie Providence, B. Zou, L. Wang and Y. Liang. "Computer vision-based Image segmentation for Fire/Smoke Detection", WASET, Paris-France (2011), June 24-26.

[13] H. Feng “A Self-Tuning Fuzzy Control System Design”. IFSA World Congress and 20th NAFIPS International Conference, Jaly 25-28, Vancouver, BC, (2001), pp. 209-214.

Jean Pau Dukuzumuremyi, he got his Master Degree of Engineering in Computer Application Technology trom School of Information Science and Engineering at Central South University, China. He is currently a Ph.D. candidate at the same University. His research interests include Digital Image Processing, Computer Graphics, Computer Vision, and their applications.

Beiji Zou, he holds a Ph.D in Computer science and technology from Hunan University,
China. South University, China. Since his graduation, Mr. Zou has been engaged in research of Digital Image Processing and Graphics. His papers were published in different Journal including Research and Development of Computer, Journal of Computer, Journal of Electronics these was presented at the International Conference sponsored by IEEE. In 1986, he participated in a project "3D Free-Form Surface Modeling", which was supported by the youth award research funds from Chinese Academy of Science.

He worked in Zhejiang University, China as a visiting scholar from Jul.1999 to Jun. 2000 and in Tsinghua University, China as a post-doctor from Jan. 2002 to Nov. 2003 respectively, 
engaged in research about Human Facial Expressional Recognition and Animation. He worked in the research field of Multimedia Technology in Griffith University of Australia from Dec. of 2003 to Dec. of 2004.

In recent years, he has focused on computer graphics, computer animation, multimedia technology and virtual reality technique. He has published sixties of papers and accomplished more than 10 projects

Damien Hanyurwimfura, he received his Master's degree of Engineering in Computer Science and Technology from Hunan University in 2010. He is currently a Ph.D student at the college of Information Science and Engineering, Hunan University, China. He is also a Lecturer at the College of Science and Technology, University of Rwanda, Kigali, Rwanda. His research interests include data security and data mining.

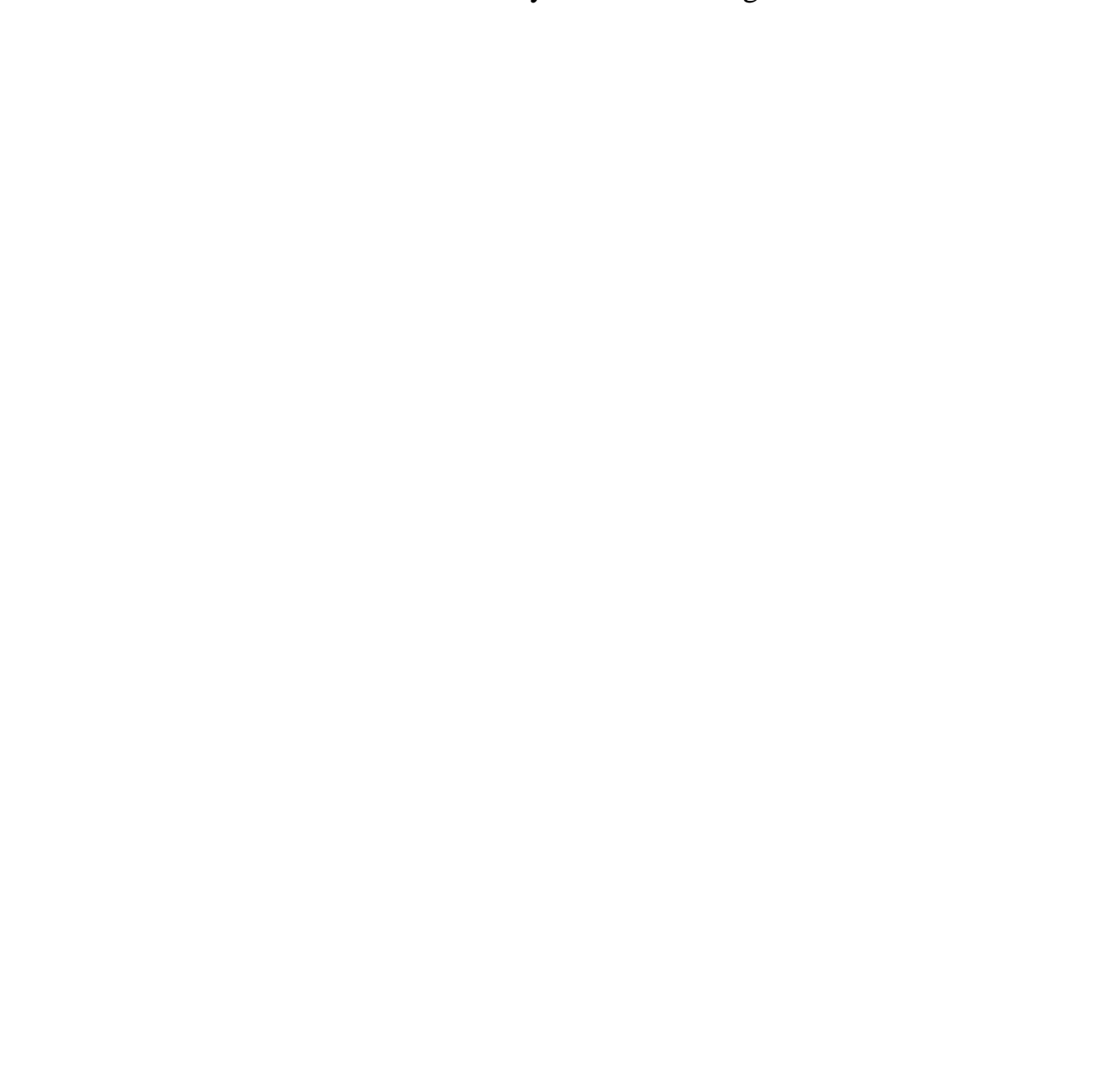


International Journal of Hybrid Information Technology

Vol.7, No.3 (2014)

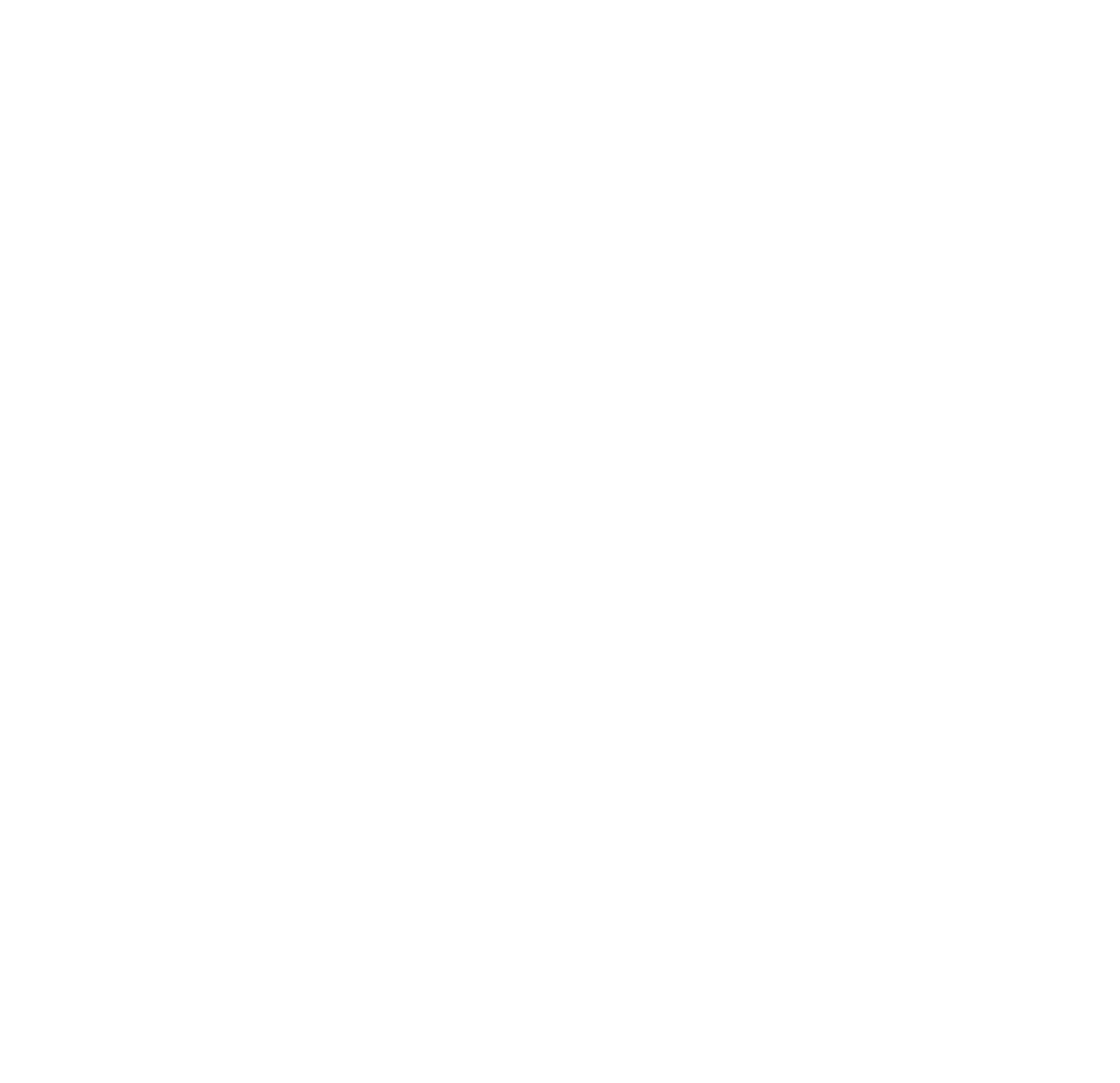

